\title{
Self-consistent numerical dispersion relation of the ablative Rayleigh-Taylor instability of double ablation fronts in inertial confinement fusion
}

\author{
C. Yañez, ${ }^{1,2}$ J. Sanz, ${ }^{2}$ and M. Olazabal-Loumé ${ }^{1}$ \\ ${ }^{1}$ CEA, CNRS, CELIA (Centre Lasers Intenses et Applications), University of Bordeaux, UMR5107, \\ F-33400 Talence, France \\ ${ }^{2}$ ETSI Aeronáuticos, Universidad Politécnica de Madrid, Madrid 28040, Spain
}

(Received 13 April 2012; accepted 15 May 2012; published online 20 June 2012)

\begin{abstract}
The linear stability analysis of accelerated double ablation fronts is carried out numerically with a self-consistent approach. Accurate hydrodynamic profiles are taken into account in the theoretical model by means of a fitting parameters method using 1D simulation results. Numerical dispersion relation is compared to an analytical sharp boundary model [Yañez et al., Phys. Plasmas 18, 052701 (2011)] showing an excellent agreement for the radiation dominated regime of very steep ablation fronts, and the stabilization due to smooth profiles. 2D simulations are presented to validate the numerical self-consistent theory. (C) 2012 American Institute of Physics.

[http://dx.doi.org/10.1063/1.4729725]
\end{abstract}

\section{INTRODUCTION}

The Rayleigh-Taylor (RT) instability is a major issue in inertial confinement fusion (ICF) capable to prevent appropriate pellet implosions. ${ }^{1}$ In the direct-drive approach, the energy deposited by directed laser irradiation ablates off the external shell of the capsule (ablator) into low-density expanding plasma. This induces a high pressure around the ablating target surface (ablation region) that accelerates the pellet radially inwards. This situation, a low density fluid pushing and accelerating a higher density one, is the standard situation for the development of RT instability, and therefore a potential source of target compression degradation.

The choice of the ablator material that provides the best performances to achieve successful implosions has been the object of intense research in recent years. First experiences were performed using hydrogenic ablators, i.e., cryogenic deuterium and tritium (DT) with a thin plastic $(\mathrm{CH})$ overcoat. The use of hydrogenic ablators is motivated by their relatively low density that permits them to achieve high ablation velocities with low in-flight aspect ratio and, therefore, exhibit good hydrodynamic stability. ${ }^{2}$ However, direct-drive cryogenic implosion experiments on the OMEGA laser facility have shown that this type of ablators presents a low threshold for the two-plasmon decay (TPD) instability leading to elevated levels of hot electron preheat for ignitionrelevant laser intensities of $10^{15} \mathrm{~W} / \mathrm{cm}^{2}$ and $351 \mathrm{~nm}$ wavelength. ${ }^{3}$ This excessive preheat is another source of compression degradation and implies not achieving the onset of ignition requirements on high total area densities and high hot spot temperatures. If hydrogenic ablators (low-Z material) are excluded as viable ablators, other concepts of target design need to be explored. One of these alternative target designs involves the use of moderate- $\mathrm{Z}$ ablators such as $\mathrm{SiO}_{2}$ or doped plastic. Recently, the performance of this concept was tested on direct-drive implosion experiments on OMEGA. ${ }^{4}$ In that study, the use glass ablators $\left(\mathrm{SiO}_{2}\right)$ suggested a mitigation of target preheat for ignition-relevant laser intensities. Thus, moderate- $Z$ materials are less affected by the TPD instability, and hence they are a potential candidate for ICF target ablators. Furthermore, experiments carried out in GEKKO XII laser facility indicated that the use of brominated plastic foils significantly reduces the growth of the RT instability compared to undoped plastic targets. ${ }^{5}$ This improvement in the hydrodynamic stability properties seems to be explained by the increasing importance of radiative energy transport in the ablated moderate- $Z$ material.

For moderate- $Z$ materials, the hydrodynamic structure of the ablation region formed by the irradiation of high intensity laser beams differs from that of low-Z materials (hydrogenic ablators). In particular, the role played by the radiative energy flux $\left(S_{r}\right)$ becomes non-negligible for increasing atomic number material and ended up forming a second ablation front. This structure of two separated ablation fronts, called double ablation (DA) front, was confirmed in the simulations carried out in Ref. 5. A qualitative measure of the relative importance of radiative and material energy transport is given by the dimensionless Boltzmann number $\mathrm{Bo}=\frac{5}{2} P \mathrm{v} / \sigma T^{4}$, where $\sigma$ is the Stefan-Boltzmann constant. A $1 D$ hydrodynamic radiation theory, ${ }^{6}$ in agreement with simulations, ${ }^{7}$ showed that below a critical value, Bo* ${ }^{*}$, of the Boltzmann number evaluated at the peak density $\left(y=y_{a}\right)$, a second minimum of the density $(\rho)$ gradient scale length $\left(|d \log \rho / d y|^{-1}\right)$ appears in the ablation region. This indicates the formation of a second ablation front at $y=y_{e}$, around the same place where radiation and matter temperatures are equal. Moreover, as the Boltzmann number decreases below Bo*, a plateau in density/temperature develops between the two fronts. In this configuration, the energy flux in the region $y_{a}<y<y_{e}$ is practically radiation dominated. Thus, the first/inner ablation front is also called, hereafter, radiation ablation (RA) front. Around the second ablation front, a transition layer (TL) develops where radiative energy flux changes its sign. This ablation front is always driven by the electronic heat flux $\left(q_{e}\right)$. However, the developed TL is as 
well a very strong emitter of radiation. Thus, in this paper, second/outer ablation front is also called electronic-radiative ablation (ERA) front. Beyond the ERA front, almost all the incoming electron heat flux is outwardly radiated $\left(q_{e}+S_{r} \approx 0\right){ }^{6}$

Recently, a first approach to a DA front linear theory of ablative RT instability has been reported. ${ }^{8}$ It deals with very steep ablation fronts that enable the use of the surface discontinuity approximation for representing both ablation fronts. It is already known from the single-ablation front linear stability theory that a sharp boundary model (SBM) requires some additional information about the flow structure behind the ablation front that cannot be introduced selfconsistently. This closure assumption has been usually considered to be the reason for its failure in reproducing numerical results. A breakthrough for SBM was given by two major considerations. ${ }^{9}$ First, the recognition of the ablation front to be an isotherm, which is actually a correct boundary condition of the problem; and second, an adequate introduction of the characteristic length of the energy deposition mechanism driving the ablation, which allows to estimate the density jump by using a simple coronal model (both implicitly contained in the analytical self-consistent models of Refs. 10 and 11). In the case of the DA front sharp boundary model, the two ablation fronts are assumed to be an isotherm, and the main properties of the ablative corona are supplied selfconsistently. Indeed, a preceding self-consistent analysis of an isolated ERA front provides the necessary information of the near-corona region structure. In particular, expressions of the perturbed mass and momentum fluxes behind the ERA front allow the fulfillment of conservation laws. Nonetheless, any stability analysis with the sharp boundary model lies on the assumption of a zero-thickness ablation front that separates two incompressible fluids at both sides of it. For single ablation fronts, this implies the study of very steep fronts, that is to say, the condition $k L_{0} \ll 1$, where $L_{0}$ is the characteristic length of the ablation front and $k$ the wavenumber of the perturbation, shall be fulfilled. However, for double ablation fronts, the picture gets complicated and it becomes also necessary to pay attention to the plateau length, $d_{p}$, the distance between the minimum density gradient scale length of the inner ablation front and the point of transition temperature. This length represents approximately the separation between fronts, i.e., the width of the DA front structure. Actually, in order to analyze the stability of DA fronts with the sharp boundary model, ${ }^{8}$ one needs to guarantee that the assumption of homogeneous fluid in the plateau region is a proper representation of the physical problem. This results in a second condition $d_{p} / L_{0} \gg 1$, which applies for both fronts. Targets with moderate- $\mathrm{Z}$ ablators such as glass ones $\left(\mathrm{SiO}_{2}\right)$ generally meet both conditions. On the contrary, for materials that radiate less, like doped plastics $(\mathrm{CHBr}, \mathrm{CHSi}, \ldots)$, we find plateau lengths of the order of the characteristic length of the inner ablation front (the outer one is usually steeper). In these cases, we should turn to self-consistent models to incorporate more accurate equilibrium profiles in the stability analysis. Self-consistent stability models start from studying the temperature and density profiles in the ablation front region, for next imposing over those profiles linear perturbations, which are analyzed, numerically ${ }^{12}$ or analytically, ${ }^{10,11}$ in order to complete the stability analysis. An analytic solution of these models is only tractable for simple situations, and, in general, we will solve them numerically. They provide better agreement with simulations, despite the difficulty for extracting physical interpretations.

The aim of this paper is to extend the DA front linear theory of ablative RT instability to those cases not considered in the sharp boundary model. We present hereafter a numerical self-consistent model to compute the growth rate of perturbations with accurate equilibrium profiles adjusted to the numerical simulations output. A similar procedure was carried out by Betti et al. but for single-ablation fronts. ${ }^{13}$ Thus, this paper is organized as follows. In Sec. II, we review the 1D hydrodynamic radiation theory and present a method to match analytical equilibrium profiles to 1D simulations ones by parameters adjustment. We study in Sec. III, the associated perturbed flow and describe the self-consistent procedure for the dispersion relation calculation. Moreover, results are compared with the existing sharp boundary model and also with new 2D numerical simulations performed with the CHIC code. ${ }^{14}$ Finally, Sec. IV is devoted to conclusions and perspectives of further work.

\section{BASE FLOW}

In the present model, we use a simplification of an existing 1D hydrodynamic radiation theory ${ }^{6}$ as the background flow. This model is suitable to describe a thin layer encompassing the ablation region, where the flow is subsonic. Thus, within this layer, the variations of the mean pressure respect to the spatial coordinate are negligible, leading to a uniform pressure that will be called hereafter $P_{0}$, the ablation pressure. Besides, it let us neglect the kinetic energy compared to the enthalpy term in the energy balance. We have then a subsonic and steady ablation region resulting from an incoming heat flow, an ablated mass flow, and an uniform pressure $P_{0}$. Two energy transport mechanisms are taken into account: the electronic heat flux and radiation. In order to perform the simplification in the base flow model that allows us to deal with the stability analysis, two asymptotic limits in the order of magnitude of the photon mean free path $\left(l_{m f}\right)$ are assumed leading to two different radiative regimes of the matter:

- Optically thin regime. This regime covers the outer ablation front and the close corona region. It is considered that the mean opacity of the plasma $\left(K \sim l_{m f p}^{-1}\right)$ is very small. This implies a gradient of the radiation energy density practically null, and therefore the radiation temperature is constant and equal to a transition temperature $T_{t}$. Thus, the radiation term in the energy conservation equation corresponds to a radiative cooling law.

- Optically thick regime. This regime covers the inner ablation front and the plateau region and it is characterized by a very large mean opacity of the plasma. In this case, a finite divergence of the radiation energy flux implies that radiation and matter are almost in equilibrium, and therefore electron temperature equals the radiation temperature, 
$T_{e}=T_{\text {rad }}=T$. This results in a radiation energy flux behaving as a thermal conduction heat flow $\left(\vec{S}_{r} \sim \nabla T\right)$.

This simplification lays on the existence of some characteristic transition temperature of the material, $T_{t}$, where the dependence of the isobaric mean opacity with the temperature changes abruptly. ${ }^{6}$

General hydrodynamic equations in the isobaric approximation are

$$
\begin{aligned}
\partial_{t} \rho+\nabla \cdot(\rho \overrightarrow{\mathrm{v}}) & =0, \quad \rho \partial_{t} \overrightarrow{\mathrm{v}}+\rho(\overrightarrow{\mathrm{v}} \cdot \nabla) \overrightarrow{\mathrm{v}}=-\nabla p+\rho \vec{g}, \\
\nabla \cdot\left(\frac{5}{2} P_{0} \overrightarrow{\mathrm{v}}-\kappa \nabla T+\vec{S}_{r}\right) & =0, \quad\left\{\begin{array}{lll}
\vec{S}_{r}=-\kappa_{R} \nabla T & \text { for } & T<T_{t}, \\
\nabla \cdot \vec{S}_{r}=4 \sigma K_{P}\left(T^{4}-T_{t}^{4}\right) & \text { for } & T \geq T_{t},
\end{array}\right.
\end{aligned}
$$

where $\kappa_{R}=\bar{\kappa}_{R} T^{\nu}$ and $\kappa=\bar{\kappa} T^{5 / 2}$ are the Rosseland and Spitzer conductivities. Equations (1) involve two differentspectral average absorption coefficients, the Planck mean opacity $K_{P} \sim l_{P}^{-1}$ and the Rosseland mean opacity $K_{R}=16 \sigma T^{3} / 3 \kappa_{R} \sim l_{R}^{-1}$. In the isobaric assumption, both opacities will only depend on the matter temperature. Moreover, the Planck mean opacity can be described as $K_{P}=$ $\bar{K}_{P} T^{-11 / 2}$ for a fully ionized plasma ${ }^{15}$ and, it is convenient to note that the frame of reference is fixed to the ablation region (to both ablation fronts since the plateau length is considered a constant). Therefore, the unperturbed velocity $\vec{v}=v_{0} \vec{e}_{y}$ corresponds to the ablation velocity of the expanding plasma.

Stationary one-dimensional profiles come from the integration of the equations

$$
\begin{aligned}
& \mathrm{v}_{0} / \mathrm{v}_{t}=\rho_{t} / \rho_{0}=\theta_{0}, \\
& \left(\theta_{0}-\left(\theta_{0}^{5 / 2}+\Delta \theta_{0}^{\nu}\right) \theta_{0}^{\prime}\right)^{\prime}=0 \quad \text { for } \quad \theta_{0}<1 \text {, } \\
& \left(\theta_{0}-\theta_{0}^{5 / 2} \theta_{0}^{\prime}\right)^{\prime}=-\beta_{t} \theta_{0}^{-11 / 2}\left(\theta_{0}^{4}-1\right) \text { for } \theta_{0} \geq 1 \text {, }
\end{aligned}
$$

with the boundary condition

$$
\theta_{0}^{\prime}=\frac{\theta_{0}-r_{D}}{r_{D}^{5 / 2}+\Delta r_{D}^{\nu}} \quad \text { when } \quad \theta_{0} \rightarrow r_{D}
$$

where $\theta_{0}=T_{0} / T_{t}$ is the dimensionless temperature, $r_{D}$ is the ratio between density at the transition temperature and the peak density $\left(r_{D} \equiv \rho_{t} / \rho_{a}<1\right), \nu$ is the power index of the Rosseland-like radiative conductivity, $\Delta=\bar{\kappa}_{R t} / \bar{\kappa}_{t}, \beta_{t}=$ $16\left(\sigma T_{t}^{4} K_{P t}\right)\left(\kappa_{t} T_{t}\right) /\left(5 P_{0} \mathrm{v}_{t}\right)^{2}$ and the prime denotes derivative respect to the spatial coordinate $\eta=y / L_{S t}$ normalized with the Spitzer length at the transition temperature, $L_{S t} \equiv 2 \kappa_{t} T_{t} /\left(5 P_{t} \mathrm{v}_{t}\right)$.

The system of Eqs. (2) provides a wide range of solutions. This variety of flow types can be explained by the presence of three different characteristic lengths in the problem. ${ }^{6}$ These characteristic lengths are associated with the different energy mechanisms that are considered. In particular, two of them related to the radiation, these are the Rosseland and Planck photon mean free paths, and the last one, the Spitzer length $L_{S}$, related to the electron thermal diffusion. In the dimensionless system of Eqs. (2), the characterization of the flow is reduced to four parameters: $r_{D}, \nu, \Delta$, and $\beta_{t}$. It is then useful to see the parameters $\Delta$ and $\beta_{t}$ as the local values, at the transition temperature, of the ratios between the characteristic lengths. That is,

$$
\Delta=\left.\frac{1}{\mathrm{Bo}} \frac{l_{R}}{L_{S}}\right|_{t}, \quad \beta_{t}=\left.\frac{1}{\mathrm{Bo}} \frac{L_{S}}{l_{P}}\right|_{t} .
$$

\section{A. Matching theoretical base flow profiles to numerical simulation ones}

Hydrodynamic stability analysis is rather sensitive to the shape of the density/temperature profiles. Actually, it is necessary to ensure that the linearization is performed around the right equilibrium profiles, that is to say, perturbed quantities shall be imposed over a base flow that can be found in the nature. In the model described above, there are four free parameters $\left(r_{D}, \nu, \Delta\right.$, and $\left.\beta_{t}\right)$ but not every combination of them is allowed. We will adjust these parameters in order to get a trustworthy background flow. These realistic equilibrium profiles are assumed to come from one-dimensional simulations. For this purpose, we use a Lagrangian hydrodynamic code, $\mathrm{CHIC},{ }^{14}$ developed at CELIA. This code includes the ion and electron heat conduction, the thermal coupling of electrons and ions and a multi-group diffusion model for the radiation transport with LTE opacities. Among different options, the following ones were retained to perform the simulations: (i) flux-limited Spitzer electron heat conduction; (ii) SESAME and QEOS equation of state; and (iii) the Thomas-Fermi ionization model.

Parameters that describe the inner ablation front $(\nu$ and $\Delta$ ) are not independent, but, instead, the plateau length (simulation output) imposes a constraint between them. In sharp boundary models, the plateau length is unambiguously defined as the distance between the two surface discontinuities. However, we have several possibilities when ablation fronts are accounted with a characteristic length. So, we define the plateau length $\left(d_{P}\right)$ as the distance between the minimum of the density gradient scale length at the RA front and the transition temperature that is estimated as the point where radiation and matter temperatures are equal. We can relate this distance with the parameters $\nu$ and $\Delta$ in the following way. Under the assumption of radiation-dominated flow at the peak density $\left(\rho_{a}\right)$, we can integrate once the energy equation of the inner front and arrive to $\theta_{0}-\Delta \theta_{0}^{\nu} \theta_{0}^{\prime} \simeq r_{D}$. It is possible to determine straightaway the point of the profile, $\theta_{0}^{*}$, where the characteristic length $L_{\text {min }}^{R A}$ takes place by setting $\left(\theta_{0} / \theta_{0}^{\prime}\right)^{\prime}=0$. This leads to $\theta_{0}^{*}=r_{D}(\nu+1) / \nu$. Then, integrating for a second time, the energy equation between $\theta_{0}=\theta_{0}^{*}$ and $\theta_{0}=1$, i.e., all along 
the plateau region, enables us to relate the plateau length to the parameter $\Delta$. This relation reads

$$
\Delta\left(\nu, r_{D}\right)=\frac{d_{P}}{L_{S t}} \varphi^{-1}
$$

where $\varphi$ is the definite integral $\int_{\theta_{0}^{*}}^{1} \theta_{0}^{\nu}\left(\theta_{0}-r_{D}\right)^{-1} d \theta_{0}$. Simple expressions can be found depending on asymptotic values of $\nu$. Actually, in the case where $\nu \gg 1$, we arrive to $\Delta \simeq$ $\nu\left(1-r_{D}\right) d_{P} / L_{S t}$ and for $\nu \ll 1, \Delta \simeq \ln \left(\nu\left(1-r_{D}\right) / r_{D}\right) d_{P}$ $/ L_{S t}$. In this way, fitting procedure is reduced to determining one parameter for each ablation front $\left(\nu\right.$ and $\beta_{t}$ for the inner and outer one, respectively). Two methods are described hereafter: an error minimization method and a gradient method.

In the error minimization method, similarly to the Betti et al. procedure for single ablation fronts, ${ }^{13}$ we define an integrated quadratic error function that, for the inner ablation fronts, reads

$$
\operatorname{err}(\nu)=\int_{\eta_{\max }}^{\eta_{t}}\left(\bar{\rho}_{s}(\eta)-\bar{\rho}_{0}(\eta)\right)^{2} d \eta
$$

where $\bar{\rho}_{s}(\eta)$ is the unperturbed density profile obtained with the simulations (normalized with the maximum density) and $\bar{\rho}_{0}(\eta)=1 / \theta_{0}(\eta)$ is dimensionless density profile given by Eqs. (2). Note that the domain of integration is from the peak density to the transition temperature. The minimization of $\operatorname{err}(\nu)$ is obtained by setting to zero the derivative respect to $\nu$,

$$
\frac{d \operatorname{err}(\nu)}{d \nu}=0
$$

This procedure can be applied analogously to the outer ablation front for the determination of $\beta_{t}$, apart from the fitting region that goes from the transition temperature to an arbitrary minimum density point (e.g., $\left.\bar{\rho}_{0}\left(\eta_{\min }\right)=0.05\right)$.

The gradient method does not need the complete simulated profiles, but two characteristic lengths computed from the $1 \mathrm{D}$ simulations output. They are the minimum gradient scale length $\left(|d \log \rho / d y|^{-1}\right)$ of the ablation front, $L_{\text {min }}^{R A}$ and $L_{\min }^{E R A}$ for the inner and outer front, respectively. This method, although less accurate than the first one, gives a good estimate of the parameters for smooth ablation fronts. It provides simple algebraic expressions by relating the parameters to the characteristic lengths. First expression is given by introducing the value of $\theta_{0}^{*}$ in the definition of $L_{\mathrm{min}}^{R A}$. It reads

$$
L_{\min }^{R A} / L_{S t}=\Delta \frac{(\nu+1)^{\nu+1}}{\nu^{\nu}} r_{D}^{\nu}
$$

For the second expression, we need to turn to a change of variable. Re-writing the optically thin energy equation with the temperature as the independent variable reads $z\left(1-5 \theta_{0}^{3 / 2} z / 2-\theta_{0}^{5 / 2} d z / d \theta_{0}\right) \simeq-\beta_{t} \theta_{0}^{-3 / 2}$, where $z \equiv \theta_{0}^{\prime}$ is a $\theta_{0}$-function. Searching the minimum of the density gradient scale length $\left(L^{E R A} \equiv \theta_{0} / z\right)$ implies $z^{\prime}=z / \theta_{0}$ and, therefore, the characteristic length can be written as
TABLE I. Parameters of the 1D analytical model that reproduces simulated hydro-profiles at time $\mathrm{t} \approx 1.8 \mathrm{~ns}$ for different ablator materials. Parameters are computed with the error minimization method.

\begin{tabular}{lcccccc}
\hline \hline & $\mathrm{CHSi}_{9 \%}$ & $\mathrm{CHSi}_{5.5 \%}$ & $\mathrm{CHBr}_{4.2 \%}$ & $\mathrm{CHBr}_{3.3 \%}$ & $\mathrm{CHBr}_{2 \%}$ & $\mathrm{SiO}_{2}$ \\
\hline$\left\langle r_{D}\right\rangle$ & 0.25 & 0.24 & 0.27 & 0.25 & 0.24 & 0.13 \\
$\langle\nu\rangle$ & 1.33 & 1.29 & 1.12 & 1.08 & 1.02 & 4.3 \\
$\left\langle\Delta / L_{S t}\right\rangle$ & 3.9 & 3.7 & 3.0 & 2.55 & 2.23 & 37.1 \\
$\left\langle\beta_{t}\right\rangle$ & 4.5 & 3.2 & 12.2 & 9.7 & 6.3 & 18.0 \\
\hline \hline
\end{tabular}

$$
L_{\min }^{E R A} / L_{S t}=\frac{7\left(\theta_{0}^{* *}\right)^{5 / 2}}{1+\sqrt{1+14 \beta_{t}}} \simeq 4.68 \beta_{t}^{-1 / 2}
$$

where $\theta_{0}^{* *}$ is the temperature at the point of minimum density gradient scale length in the outer ablation front $\left(\theta_{0}^{* *} \approx(5 / 2)^{2 / 5}\right)$.

Equations (5)-(9) give us the set of parameters that provide realistic hydrodynamic profiles. Table I presents some results of $1 \mathrm{D}$ CHIC simulations with the corresponding parameters calculated with the error minimization method. We considered a $25 \mu \mathrm{m}$ thick ablator in planar geometry that is irradiated by a $3 \mathrm{~ns}$ constant laser pulse of $100 \mathrm{TW} / \mathrm{cm}^{2}$. Initial density for the doped plastic layers is obtained from the formula $\rho_{D}=\rho_{U} \sum_{i} A_{i} F_{i} /\left(\sum_{i} A_{i} F_{i}\right)_{U}$, where $\rho_{D}$ and $\rho_{U}$ are the density of the doped and undoped material, respectively, $A_{i}$ is the mass number and $F_{i}$ is the atom fraction for each element. $^{16}$ In our case that expression gets reduced to $\rho_{D}=\rho_{C H}\left(A_{C H} F_{C H}+A_{d o p} F_{d o p}\right) /\left(A_{C H} F_{C H}\right)=\rho_{C H} /(1-x)$, where $x$ is the mass fraction of the dopant. A practically constant acceleration is observed from $t \approx 1.5 \mathrm{~ns}$, and values of the analytical parameters are given for the hydro-profiles at $t \approx 1.8 \mathrm{~ns}$.

A relevant feature of the density/temperature profiles is given by the ratio $L_{\min }^{R A} / d_{p}$ (see Table II). We can classify the different ablator materials, for which the DA front appear, into two groups (Refs. 6 and 7): weakly radiant fronts where the ratio is of the order of unity, and strongly radiant ones where $L_{\min }^{R A} / d_{p} \ll 1$. The glass ablators belong to the latter case. In the acceleration stage, the hydrodynamic profiles show two steep ablation fronts clearly separated by a plateau region. Sharp boundary models are then suitable to analyze the stability of the fluid structure. On the contrary, doped plastic ablators present profiles where the plateau region is not distinguished from the inner ablation front (their characteristic length is of the same order). In this case, a density/ temperature profile composed of layers of homogeneous flows separated by discontinuity surfaces is no longer a proper representation of the problem, and sharp boundary

TABLE II. Initial density, mean minimum density gradient scale length of the radiative, and the electron-radiative ablation fronts in the interval $1.5 \mathrm{~ns}<\mathrm{t}<2.5 \mathrm{~ns}$ for different ablator materials.

\begin{tabular}{lllccr}
\hline \hline & $\rho_{i}(\mathrm{~g} / \mathrm{cc})$ & $\left\langle r_{D}\right\rangle$ & $\left\langle L_{\text {min }}^{R A} / L_{S t}\right\rangle$ & $\left\langle L_{\text {min }}^{E R A} / L_{S t}\right\rangle$ & $\left\langle d_{p} / L_{S t}\right\rangle$ \\
\hline $\mathrm{CHSi}_{9 \%}$ & 1.50 & 0.27 & 4.83 & 1.94 & 5.24 \\
$\mathrm{CHBr}_{4.2 \%}$ & 1.62 & 0.21 & 4.70 & 1.24 & 4.56 \\
$\mathrm{SiO}_{2}$ & 2.2 & 0.12 & 3.50 & 1.04 & 13.23 \\
\hline \hline
\end{tabular}




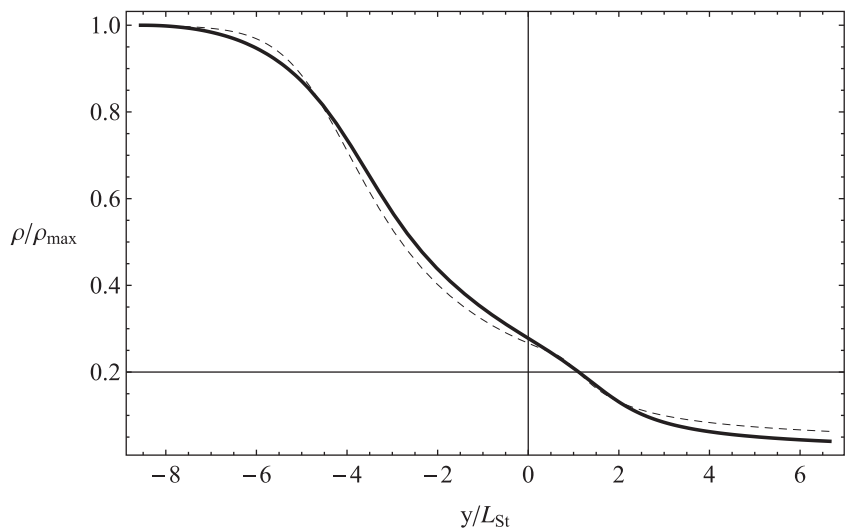

FIG. 1. Dimensionless density profile taken from CHIC simulations (solid line) with ablator material $\mathrm{CHSi}_{9 \%}$ at time $t \simeq 1.8 \mathrm{~ns}$, compared to the density profile computed from the model (dashed line) with the parameters $r_{D}=0.25, \beta_{t}=4.5, \nu=1.33$, and $\Delta / \mathrm{L}_{\mathrm{St}}=3.9$.

models are expected to fail in giving an accurate approximation of the Rayleigh-Taylor growth rate. This supports the necessity of having a self-consistent model for stability studies. In Figs. 1 and 2, we show comparisons between profiles computed from the theory and those of the simulations. In both cases, we use doped plastic as the ablator material (one with the bromine dopant and the other with the silicon one), and a remarkable agreement is found.

We focus now our attention on the value of the parameter $\nu$. The Rosseland thermal conductivity is defined as $\kappa_{R}=16 \sigma T^{3} / 3 K_{R}=\bar{\kappa}_{R} T^{\nu}$. The dependence on the temperature comes from two factors: explicitly from the cubic factor and implicitly from the Rosseland mean opacity. For an ionized material, opacity is a decreasing function of the temperature, that is to say, it becomes more transparent as the temperature increases. A simple model describing the opacity was proposed in Ref. 6 and consists of a piecewise continuous power law of temperature in the way

$$
K_{R}= \begin{cases}\bar{K}_{R}\left(T / T_{a}\right)^{-q_{1}}, & T<T_{t}, \\ \bar{K}_{R}\left(T_{t} / T_{a}\right)^{-q_{1}}\left(T / T_{t}\right)^{-q_{2}}, & T \geq T_{t},\end{cases}
$$

where $\bar{K}_{R}$ is the Rosseland mean opacity at the peak density $\rho_{a}$ and temperature $T_{a}$, and $0<q_{1}<q_{2}$. Thus, we expect to have $\nu>3$, which is not the case. A possible explanation of

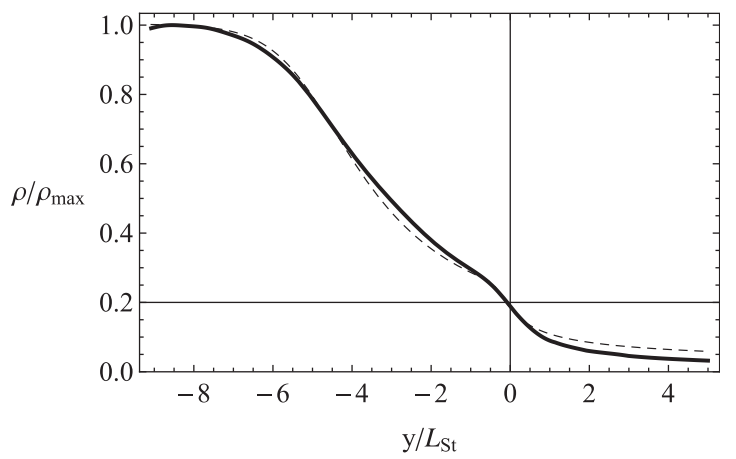

FIG. 2. Dimensionless density profile taken from CHIC simulations (solid line) with ablator material $\mathrm{CHBr}_{4.2 \%}$ at time $t \simeq 1.8 \mathrm{ns,} \mathrm{compared} \mathrm{to} \mathrm{the} \mathrm{den-}$ sity profile computed from the model (dashed model) with the parameters $\mathrm{r}_{\mathrm{D}}=0.27, \beta_{t}=12.2, \nu=1.12$, and $\Delta / \mathrm{L}_{\mathrm{St}}=3$.

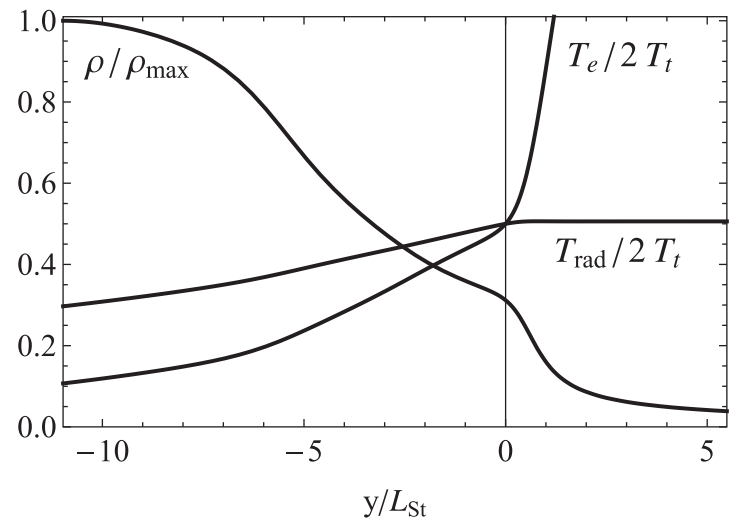

FIG. 3. Dimensionless profiles of density, electron temperature, and radiation temperature taken from CHIC simulations with ablator material $\mathrm{CHBr}_{4.2 \%}$ at time $\mathrm{t}=2.0 \mathrm{~ns}$.

this divergence between the one-dimensional radiation hydrodynamic model and results derived from the simulations can be noticed in Fig. 3. We can see that immediately beyond the transition temperature (approximately the point where the matter and radiation temperatures are equal), the matter becomes transparent as a consequence of the sharp increase of the electron temperature. This yields to a radiation temperature that remains constant, which is a main property of the optically thin regime. On the contrary, the assumption of an optically thick regime for $T<T_{t}$ is not fully satisfied, since radiation and electron temperatures do not stay the same. Nevertheless, the adjustment of parameters from simulations output let us partially recover some of the missing physics regarding the radiative ablation front, included in the low values of the power index $\nu$.

Another source of discrepancy between the onedimensional theory and the simulations comes from the assumption of a diffusion grey model for the radiation energy flux $S_{r} \cdot{ }^{17}$ That model assumes a Planck's spectrum $U_{v}(\vec{r}, t) \approx U_{P_{v}}\left(T_{r a d}(\vec{r}, t)\right)$ for the radiation field, which enables to calculate the mean opacity as spectral averages of the absorption coefficient $\kappa$. In particular, the Planck mean opacity reads

$$
K_{P} U_{P}(T) \equiv K_{p} 4 \sigma T^{4} / c=\int_{0}^{\infty} \kappa_{v} U_{P v}(T) d v,
$$

and the Rosseland mean opacity

$$
K_{R}^{-1} d U_{P} / d T \equiv K_{R}^{-1} 16 \sigma T^{3} / c=\int_{0}^{\infty} \kappa_{v}^{-1}\left(d U_{P v} / d T\right) d v
$$

where $v$ is the frequency and $\kappa_{v}$ the absorption coefficient per frequency. However, it is known that whereas the spectral radiant energy density follows approximately a Planckian distribution in a layer around the outer ablation front, it does not close to the peak density. ${ }^{6}$ This causes an overestimate of the radiation heating $\left(\propto \nabla \cdot \vec{S}_{r}\right)$ in a layer around the inner ablation front, and consequently the analytical model fails in an accurate representation of the hydrodynamic profiles.

As commented above, a similar procedure for determining accurate hydro-profiles was already carried out in Ref. 13 
in the case of single ablation fronts. There, an error minimization method led to obtain the effective power index for thermal conduction $\nu$ and the ablation front thickness by fitting the analytic density profile to the $1 \mathrm{D}$ simulation results. Note that the power index $\nu$ used in Ref. 13 refers to a general thermal conduction (including electron and radiation effects) and not to a specific Rosseland-like radiative conduction as treated in the DA front theory. In the case of solid DT targets steep profiles were found with $\nu \approx 2$ (close to the Spitzer value) that indicate a very low level of radiation transport for this material. However, the use of a higher $\mathrm{Z}$ target material like $\mathrm{CH}$ gave smoother density profiles and a lower value of the power index $(\nu \approx 0.7)$. This shows that radiation transport becomes non-negligible in the case of plastic ablators, although the single temperature model still holds. However, this model breaks down for moderate- $Z$ ablators with the emergence of the second ablation front, making necessary to turn to a DA front theory. The influence of the material atomic number in the hydro-profiles is intrinsically connected to the relative importance of radiation transport in the ablation region, and schematically the evolution with $\mathrm{Z}$ of the density profiles passes from one steep ablation front (with very low-Z ablators, e.g., DT) to a smooth one (low-Z ablators, e.g., $\mathrm{CH}$ ) with increasing importance of radiation field. Next, this smooth front breaks into two (low/ moderate- $\mathrm{Z}$ ablators, e.g., doped $\mathrm{CH}$ ) due to the absorption of the radiation energy and electron heat fluxes at two different locations (the two ablation fronts). Finally, both ablation fronts get steeper and a plateau density region develops between them (moderate- $\mathrm{Z}$ ablators, such as $\mathrm{SiO}_{2}$ ).

\section{PERTURBED FLOW}

In the frame of a self-consistent analysis, the perturbed problem is governed by the same equations used in the study of the background flow, that is to say, in our case, the mass, momentum, and energy conservation around the ablation region (1). The stability analysis is restricted to a twodimensional domain for simplification, so we only consider flow perturbations along the $x$ and $y$ spatial coordinates. We look then for solutions of perturbed quantities in the wavelike form $\exp (\gamma t+i k x)$. Any hydrodynamic quantity is expanded to the first order perturbation, for instance $\overrightarrow{\mathrm{v}}(x, y, t)=\left(\mathrm{v}_{1 x}(y) e^{\gamma t+i k x}, \mathrm{v}_{0}(y)+\mathrm{v}_{1 y}(y) e^{\gamma t+i k x}\right)$, and the set of Eqs. (1) is linearized around the zero order flow. This leads to an eigenvalue problem that can be expressed in a matrix form as

$$
\overrightarrow{\mathrm{V}}^{\prime}= \begin{cases}\mathbf{A}^{R A} \vec{V} & \text { for } \quad r_{D}<\theta_{0}<1, \\ \mathbf{A}^{E R A} \vec{V} & \text { for } \quad \theta_{0} \geq 1,\end{cases}
$$

where $\vec{V}$ is the vector of the first order hydrodynamic quantities normalized by their base flow value at the transition temperature $\left(T_{t}, \rho_{t}, \mathrm{v}_{t}, \ldots\right)$, and the fifth order square matrix, $\mathbf{A}^{R A}$ and $\mathbf{A}^{E R A}$ (for the inner and outer ablation front regions, respectively) depend on the base flow $\left(\theta_{0}, r_{D}, \beta_{t}, \nu\right.$, and $\left.\Delta\right)$. The prime denotes a derivative respect to the independent variable $\theta_{0}$. Here, the Froude number, $\mathrm{Fr}_{\mathrm{t}}$, is defined as $\mathrm{Fr}_{\mathrm{t}}=\mathrm{v}_{t}^{2} / g L_{S t}$, and $\hat{k}=k L_{S t}$ and $\hat{\gamma}=\gamma L_{S t} \mathrm{v}_{\mathrm{t}}$ are the normal- ized wavenumber and growth rate, respectively. Notice that normalized perturbed velocities are denoted by $u_{1 x}, u_{1 y}$, the temperature by $\theta_{1}$ and the perturbed pressure by $p_{1}$. Detailed expressions can be found in Appendix A. There is one change of variables ${ }^{8}$ that is convenient to make prior to the computation in order to avoid the appearance of null eigenvectors in the modal analysis and also to increase our physical insight in the problem. Thus, perturbed quantities read

$$
\begin{aligned}
G_{1} & =\theta_{0} F_{1}=u_{1 y}-\theta_{1}, \quad W_{1}=i \hat{k} u_{1 x}, \\
Q_{1} & =2 u_{1 y}+p_{1}-\theta_{1}, \\
T_{1}^{R A} & =\left(\Delta \theta_{0}^{\nu}+\theta_{0}^{5 / 2}\right) \theta_{1}, \quad T_{1}^{E R A}=\theta_{0}^{5 / 2} \theta_{1}, \\
H_{1}^{R A} & =\left(\left(\Delta \theta_{0}^{\nu}+\theta_{0}^{5 / 2}\right) \theta_{1}\right)^{\prime}-u_{1 y}, \\
H_{1}^{E R A} & =\left(\theta_{0}^{5 / 2} \theta_{1}\right)^{\prime}-u_{1 y},
\end{aligned}
$$

which leaves the unknown vector as $\vec{Y}$ $=\left(G_{1}, W_{1}, Q_{1}, T_{1}, H_{1}\right)$, and the matrices of the fifth order system of differential equations are renamed to $\mathbf{M}^{\mathbf{R A}}$ and $\mathbf{M}^{\text {ERA }}$. Thus, the eigenvalue problem reads

$$
\begin{gathered}
\vec{Y}^{\prime}=\left\{\begin{array}{llll}
\mathbf{M}^{\mathbf{R A}} \overrightarrow{\mathrm{Y}} & \text { for } & r_{D}<\theta_{0}<1, \\
\mathbf{M}^{\mathrm{ERA}} \overrightarrow{\mathrm{Y}} & \text { for } & \theta_{0} \geq 1,
\end{array}\right. \\
\mathbf{M}^{\mathbf{R A}}=\left(\begin{array}{ccccc}
\frac{\theta_{0}^{\prime}}{\theta_{0}} & -1 & 0 & \frac{\hat{\gamma}}{\theta_{0} R\left(\theta_{0}\right)} & 0 \\
-2 \hat{k}^{2} & -\frac{\hat{\gamma}}{\theta_{0}} & \hat{k}^{2} & -\frac{\hat{k}^{2}}{R\left(\theta_{0}\right)} & 0 \\
-\frac{\hat{\gamma}}{\theta_{0}} & -1 & 0 & -\frac{F r_{t}^{-1}}{\theta_{0}^{2} R\left(\theta_{0}\right)} & 0 \\
1 & 0 & 0 & \frac{1}{R\left(\theta_{0}\right)} & 1 \\
0 & 1 & 0 & \hat{k}^{2} & 0
\end{array}\right), \\
\mathbf{M}^{\mathbf{E R A}}=\left(\begin{array}{ccccc}
\theta_{0}^{\prime} & -1 & 0 & \frac{\hat{\gamma}}{\theta_{0}^{7 / 2}} & 0 \\
-\frac{\hat{k}_{0}}{\theta_{0}} & -1 & 0 & -\frac{F r_{t}^{-1}}{\theta_{0}^{9 / 2}} & 0 \\
-\frac{\hat{\gamma}}{\theta_{0}} & \hat{k}^{2} & -\frac{\hat{k}^{2}}{\theta^{5 / 2}} & 0 \\
1 & 0 & 0 & \frac{1}{\theta_{0}^{5 / 2}} & 1 \\
0 & 1 & 0 & \hat{k}^{2}-\frac{\beta_{t}\left(3 \theta_{0}^{4}-11\right)}{2 \theta_{0}^{9}} & 0
\end{array}\right),
\end{gathered}
$$

where $R\left(\theta_{0}\right)=\Delta \theta_{0}^{\nu}+\theta_{0}^{5 / 2}$. It is worth noting the physical meaning of $F_{1}, Q_{1}$, and $H_{1}$ that represent the local flux of mass, momentum, and energy, respectively. In the above system of equations, for a given base flow and a given acceleration of the foil, the only remaining free parameters are the perturbation wavenumber and its growth in time, i.e., the dispersion relation. The statement of the linear problem is completed with the boundary conditions that provide the necessary information to close the perturbed problem, and therefore to determine the dispersion relation. 
Boundary conditions impose constraints on the perturbations; in particular, we can state that unstable disturbances in the flow shall be localized within the double ablation front region. In other words, perturbation shall vanish down- and upstream. This is equivalent to set $Y_{i} \rightarrow 0$ in both limits $\theta_{0} \rightarrow r_{D}$ (or $\eta \rightarrow-\infty$, peak density) and $\theta_{0} \rightarrow \infty(\eta \rightarrow \infty$, near-corona region). In that way, the boundary condition at the peak density determines the starting point of the integration (a linearly stable mode), whereas the boundary condition at near-corona region leads to the dispersion relation $\hat{\gamma}=\hat{\gamma}(\hat{k}$, base flow, acceleration $)$. Mathematically, we need to carry out a modal analysis of the matrices $\mathbf{M}^{\mathbf{R A} / \mathbf{E R A}}$ to be able to discriminate stable and unstable eigenmodes to properly state the boundary conditions.

At the peak density $\left(\rho_{a}=1 / r_{D}\right)$, we perform a matrix expansion around the singular point $\left(\theta_{0}-r_{D}\right)$ in order to determine the five independent eigenmodes of $\mathbf{M}^{\mathbf{R A}}{ }^{8}$ Notice that the asymptotic behavior of the temperature is $\theta_{0} \simeq r_{D}+c_{0} e^{a \eta}$, where $c_{0}$ is an arbitrary constant depending on the origin of coordinates and $a=\left(r_{D}^{5 / 2}+\Delta r_{D}^{\nu}\right)^{-1}>0$. Therefore, the leading order of the eigenmodes goes like $\left(\theta_{0}-r_{D}\right)^{\lambda} \sim e^{\lambda a \eta}$, where $\lambda$ is the associated eigenvalue. In order to fulfill the boundary condition, we are only interested in having bounded modes developing at the peak density, what, in this case, means a positive eigenvalue (since $\eta \rightarrow-\infty)$. In particular, there are two of them

$$
\lambda_{1}^{\text {left }}=\hat{k} / a, \quad \lambda_{2}^{\text {left }}=\left(1+\sqrt{1+4 \hat{k}^{2} / a^{2}+4 \hat{\gamma} /\left(a r_{D}\right)}\right) / 2
$$

and we discard the three unbounded eigenmodes.

The linearity of the problem allows us to write the starting point of the integration as a linear combination of the two stable modes in the way

$$
\vec{Y}^{l e f t}=\alpha_{1}\left(\theta_{0}-r_{D}\right)^{\lambda_{1}^{l e f t}} \vec{Y}_{1}^{l e f t}+\alpha_{2}\left(\theta_{0}-r_{D}\right)^{\lambda_{2}^{l e f t}} \vec{Y}_{2}^{l e f t}
$$

where $\alpha_{1}$ and $\alpha_{2}$ are two undefined parameters and $\vec{Y}_{1}^{l e f t}$ and $\vec{Y}_{2}^{\text {left }}$ are the eigenmodes vectors for $\lambda_{1}^{\text {left }}$ and $\lambda_{2}^{\text {left }}$, respectively.

Analogously, at the near-corona region, the boundary condition is composed of a linear combination of three bounded eigenmodes $(\lambda<0)$. However, in this case, the computation procedure (described in Sec. III A) is only concerned with the most unbounded eigenmode, so we focus on this mode that, at leading order and for $\beta_{t} \gg 1$, reads

$$
Y^{r i g h t}(x) \propto x^{2 / 5} \exp \left(\hat{k} x-2(5 / 2)^{1 / 5} \beta_{t}^{-2 / 5} x^{1 / 5}\right),
$$

where $x \equiv 2 \theta_{0}^{5 / 2} /\left(5 \beta_{t}^{1 / 2}\right)$.

A more general expression of the modal analysis can be found in Appendix B.

\section{A. Growth rate calculation}

The method used in the computation of the dispersion relation is similar to the one used by Kull in electronic ablation fronts. ${ }^{12}$ First, we need to define the base flow with the set of parameters $\left(r_{D}, \beta_{t}, \nu\right.$, and $\left.\Delta\right)$, the acceleration at which the foil is subjected to, and the wavelength of the perturbation. Next, we integrate the matrix system (15) forwards from the boundary condition $\vec{Y}^{\text {left }}$ to a distance of several perturbation wavelengths. The solution generally explodes, since the unbounded modes at the near-corona region develop; this means that the boundary condition is not satisfied in that region. The way to impose vanishing perturbations away from the ablation fronts is the following: we normalize the vector solution by the most unbounded mode (20). Thus, the solution will tend to a constant vector $\vec{C}$ when $\theta_{0} \gg 1$. Actually, $Y^{\text {right }} \vec{C}$ represents the exploding mode that shall be null to ensure a bounded solution. Linearity of the problem enables us to express each component of the vector $\vec{C}$ as a linear combination of $\alpha_{1}$ and $\alpha_{2}$ (the free parameters of the boundary condition at the peak density (19)), let it be, $\vec{C}=\vec{f}\left(\alpha_{1}, \alpha_{2}\right)=\alpha_{1} \vec{f}(1,0)+\alpha_{2} \vec{f}(0,1)$. In order to have a non-trivial solution, we select any two components $\mathrm{i}, \mathrm{j}$ of the vector solution and force the following determinant to be zero:

$$
\left|\begin{array}{ll}
f_{i}(1,0) & f_{j}(1,0) \\
f_{i}(0,1) & f_{j}(0,1)
\end{array}\right|=0
$$

which yields the growth rate of the perturbation.

\section{B. Influence of the parameter $v$ on the stability}

In this paragraph, we review the influence of the parameter $\nu$ on the stability of a single ablation front driven by thermal conduction. ${ }^{13}$ As it was explained above, the adjustment of the parameters in order to reproduce realistic flow profiles relaxes the constraint of $\nu>3$ that was imposed, in the case of radiation-dominated ablation fronts, by the 1D radiation-hydrodynamic theory. This allows us to have a wider range of $\nu$ values to consider. In the results presented within this paper (Table I), we find $\nu>1$ in all the ablator materials and laser power explored. However, an analogous study ${ }^{13}$ performed with plastic $(\mathrm{CH})$ and beryllium $(\mathrm{Be})$ targets revealed values of the power index less than the unity.

We use the mathematical procedure described in the previous paragraph with the boundary conditions and equations detailed in Appendix C. Stability analysis results for a single ablation front are summarized in Fig. 4 with the representation of the neutral curve, i.e., the cut-off wavenumber

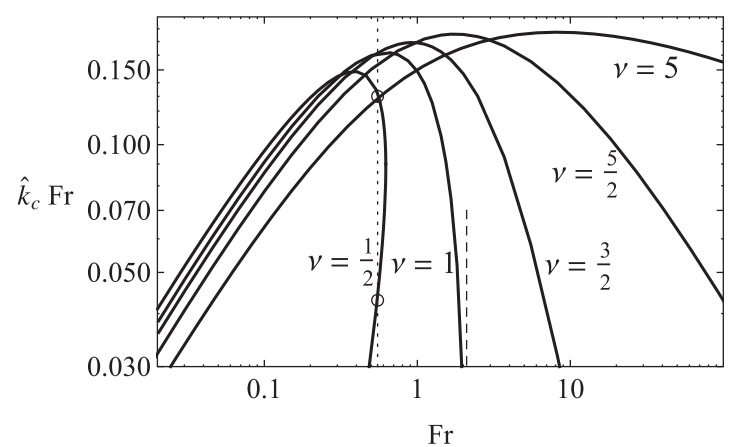

FIG. 4. Neutral curve of a single ablation front depending on the Froude number and the conductivity power index $\nu$. 
in function of the governing parameters $\nu$ and Fr. The neutral curve illustrates the border between an unstable problem (the region contained within the curve) and a stable one. Depending on the order of magnitude of the Froude number, two asymptotic behaviors are found:

- For Fr $\ll 1$, the role of an increasing value of $\nu$ is weakly stabilizing (the cutoff wavenumber gets reduced). Numerical results in this limit fit pretty well with the analytic expression given by Piriz et al. ${ }^{9}$

$$
\kappa_{c}=h(\nu) \operatorname{Fr}^{2 / 3}, \quad h(\nu) \approx 1.5 \frac{(2 \nu+2)^{(2 \nu+2) / 3}}{(2 \nu+3)^{(2 \nu+3) / 3}},
$$

where $\kappa_{c}=\hat{k}_{c} \mathrm{Fr}$ and the variables are normalized with the characteristic length and the velocity evaluated at the peak density.

- For Fr $\gg 1$, the tendency is just the opposite and the cutoff wavenumber increases for higher values of $\nu$. In this case, the range of unstable perturbation wavelengths widens for higher $\nu$. A good agreement is found with the analytic theory of Betti et al. that predicts a cut-off wavenumber. ${ }^{13}$

$\kappa_{c}=\mu(\nu) \operatorname{Fr}^{-1 /(\nu-1)}, \mu(\nu) \approx\left(\frac{(2 / \nu)^{1 / \nu}}{\Gamma(1+1 / \nu)}+\frac{0.12}{\nu^{2}}\right)^{\nu /(\nu-1)}$,

where $\Gamma(x)$ is the gamma function. Note that in this limit, the asymptotic behavior of $\hat{k}_{c}$ is a power law with an exponent dependent of $\nu$, which strongly destabilizes steep hydrodynamic profiles. This exponent dependence breaks up for $\nu=1$ (the analytic theory was built up with the constraint of values $\nu>1$ ). Nevertheless, numerically it is observed in this case a vertical asymptote in the neutral curve at $\mathrm{Fr} \approx 2$. This means that smooth hydrodynamic profiles with $\nu \simeq 1$ subjected to large Froude number $(\mathrm{Fr}>2)$ are stabilized for all wavelengths. ${ }^{13}$

In the region where $\operatorname{Fr} \sim O(1)$, it is not possible to establish a general behavior, since neutral curves get crossed (see Fig. 4).

It is also worthy to notice a peculiar feature observed in neutral curves for $\nu<1$. In this case, there exists a cut-off Froude number from which the ablation front is stable, in other words, a sufficiently small acceleration will not lead to perturbation growth. From this point of the plot, two different branches of the neutral curve develop as the Froude number decreases. This yields the appearance of two different cut-off wavenumbers, staying away from the classical picture of the ablative RT instability. Actually, instead of following the trend of unstable growth rate $\gamma=\sqrt{\mathrm{kg}}$ for very small wavenumbers, there is a stable region. Thus, dispersion relation is composed of three regions: two stable regions (for both small and large wavenumbers) and an intermediate unstable region which is delimited by two cut-off wavenumbers. An example of this behavior can be seen in Fig. 5, where we show the case $\mathrm{Fr}=0.55$ for two different values of thermal conductivity power index. The existence of the

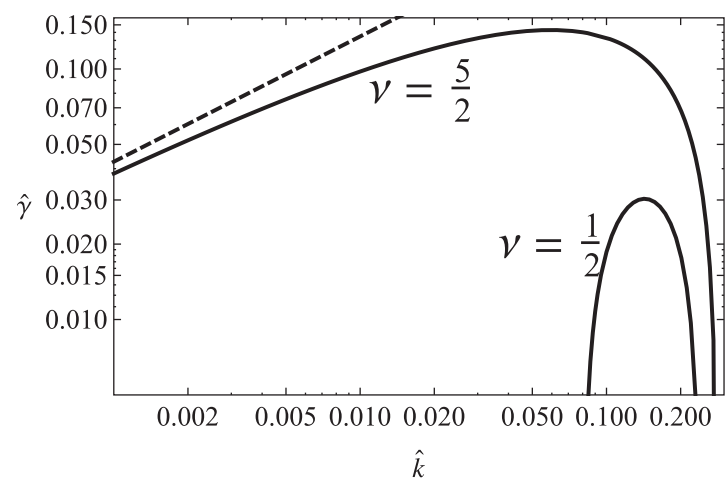

FIG. 5. Dispersion relation for two different values of the conductivity power index $\nu$ and $\mathrm{Fr}=0.55$. Dashed line represents the classical RT growth rate $\gamma=\sqrt{\mathrm{kg}}$.

cut-off for long-wavelength modes is explained by an enhanced restoring force due to the hydrodynamic pressure (rocket effect) for those modes. In a very schematic approach with $k L_{0} \ll 1 \quad\left(L_{0}=\right.$ characteristic length of the ablation front) and following Ref. 9, the leading terms of the relation dispersion for single ablation front are

$$
\gamma \simeq \sqrt{k\left(g-(\nu / 2)^{1 / \nu}\left(k L_{0}\right)^{1-1 / \nu} \mathrm{v}_{a}^{2} / \mathrm{L}_{0}\right)}-2 k \mathrm{v}_{a},
$$

where $\mathrm{v}_{a}$ is the ablation velocity. We observe two kind of stabilizing mechanisms. The term inside the square root and proportional to $\mathrm{v}_{a}^{2}$ (rocket effect) is some kind of overpressure or enhancement of the dynamic pressure occurring in the crests of the corrugated ablation front (an under pressure is occurring in the valleys). This self-regulation of the ablation pressure when the front is perturbed is related to the variation of the local temperature gradient. The second stabilization mechanism (so-called convective stabilization), the term proportional to $k \mathrm{v}_{a}$, has origin in the effects of fire polishing, mass convection, and vorticity (see Ref. 9).

The dependence of the rocket effect term on the wavenumber depends on the nature of the thermal conduction mechanism. For a general thermal conductivity $\left(\kappa \sim T^{\nu}\right)$, the rocket effect term is proportional to $k^{1-1 / \nu} \cdot{ }^{10,11,13}$ If $\nu>1$, the rocket effect can lead to complete stability for sufficiently large perturbation wave numbers. But if $\nu<1$, the rocket effect affects the small perturbation wavenumbers and can completely suppress the associated unstable modes, which explains the smallest cut-off wavenumber that we found in Figs. 4 and 5.

\section{Comparisons with the sharp boundary model and 2D CHIC simulations}

As a first test of the numerical self-consistent theory, we compare the numerical dispersion relation with the analytic formula for DA fronts. ${ }^{8}$ The latter has been developed from the surface discontinuity assumption, so we expect an agreement of the results as the characteristic scale lengths of both fronts get shorter. We also use in the comparison a simplified and much more compact dispersion relation formula derived from the analytic theory. Derivation of this easy-to-use formula is explained in Appendix D, and the basis of the 


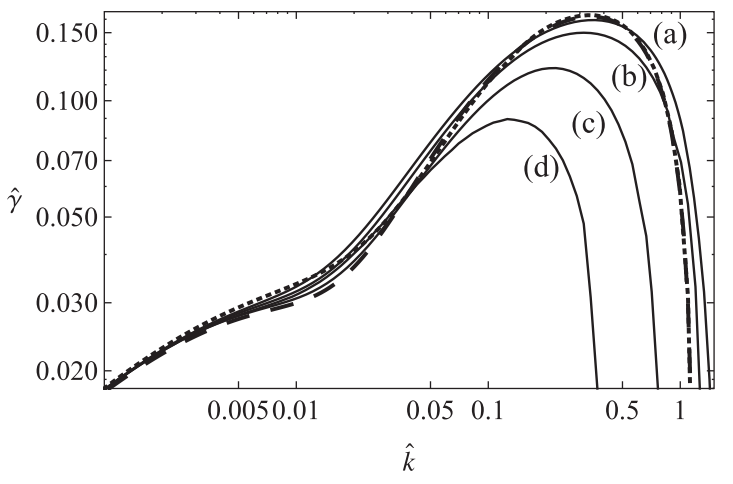

FIG. 6. Dispersion relation obtained from the numerical method (solid line) for the parameters $r_{D}=0.25, \beta_{t}=20, \mathrm{Fr}_{\mathrm{t}}=2$ and (a) $\nu=10$ and $\Delta=87$, (b) $\nu=5$ and $\Delta=41$, (c) $\nu=5 / 2$ and $\Delta=20$, and (d) $\nu=6 / 5$ and $\Delta=15$. Dashed line corresponds to the analytic formula with $d_{P}=12$ and dotted line plots the asymptotic limit of the analytic formula (see Appendix D).

simplification lies in a matching formula between two asymptotic wavelength limits: much shorter and larger than the plateau length.

Fig. 6 shows such a comparison, where we apply the numerical method to four cases varying the characteristic scale length of the RA front. In order to make comparable the different background flows, we keep constant the plateau length (in this case $d_{P} \approx 12$ ) and the density ratio, $r_{D}=0.25$. Thus, $L_{\min }^{R A}$ is controlled by the pair of parameters $\nu$ and $\Delta$, and we pass from a case (a) where the RA front can be assimilated to a surface discontinuity $\left(L_{\min }^{R A} / L_{S t} \simeq 2 \cdot 10^{-3}\right.$ and $L_{\min }^{R A} / d_{P} \simeq 2 \cdot 10^{-4}$ ) to a case (d) where the RA front is smooth and completely covers the plateau region $\left(L_{\min }^{R A} / L_{S t} \simeq 13\right.$ and $\left.L_{\min }^{R A} / d_{P} \simeq 1\right)$. Intermediate cases are (b) and (c) with $L_{\min }^{R A} / L_{S t} \simeq 0.6$ and $L_{\min }^{R A} / L_{S t} \simeq 5$, respectively. The finite characteristic length of the ablation fronts shall not influence perturbation modes of very large wavelength $\left(k L_{\min }^{R A} \ll 1\right)$, since these modes cannot distinguish the details of the hydro-profiles. Thus, as it is expected, sharp boundary model results are closer to those of case (a). In particular, important target design parameters such as maximum growth rate and the associated wavelength are in excellent agreement, and the sharp boundary model can be used as an accurate estimate. Moreover, the simplified dispersion formula also provides a remarkable agreement. The situation changes when the density is smoothly varying in the RA front and the perturbation wavelength is of the order of the minimum gradient scale length $\left(k L_{\min }^{R A} \sim O(1)\right)$. In this case, perturbed modes are affected by the profile gradient that yields to the mitigation of the unstable term for short wavelengths. In the classical theory, this is captured by the asymptotic formula

$$
\gamma_{c l}=\sqrt{\frac{A_{T} k g}{1+A_{T} k L_{\min }}}
$$

where $A_{T}=\left(\rho_{h}-\rho_{l}\right) /\left(\rho_{h}+\rho_{l}\right)$ is the Atwood number, and $\rho_{h}$ and $\rho_{l}$ are the density of the heavy and the light fluid, respectively. An analogous mitigation for short wavelength modes is observed in the ablation region stability of Fig. 6, where the cut-off wavenumber decreases as the characteristic length of the RA front increases. Furthermore, maximum growth rate is reduced by a factor of 2 from the configuration of case (a) to the one of case (d).

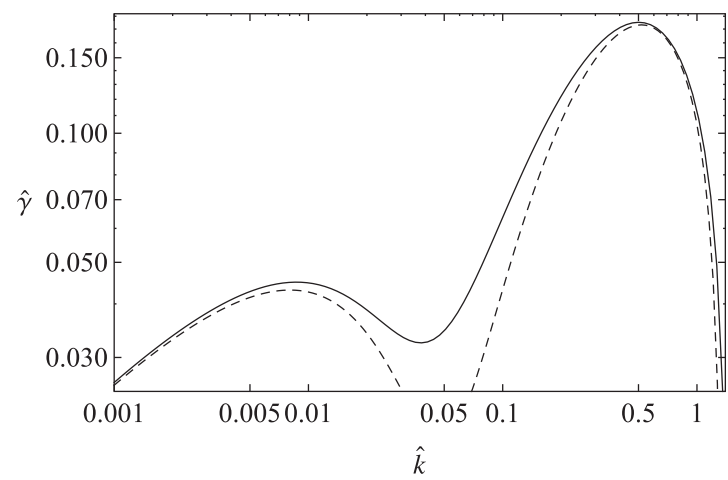

FIG. 7. Dispersion relation obtained from the numerical method (solid line) for the parameters $r_{D}=0.35, \beta_{t}=20, \mathrm{Fr}_{\mathrm{t}}=1, \nu=10$, and $\Delta=20$. Dashed line corresponds to the analytic formula with $d_{P} \approx 3$.

A peculiar feature of the dispersion relation given by the SBM is the appearance of a double-hump shape for short plateau configurations. ${ }^{8}$ In Fig. 7, we show that such a dispersion relation shape is also found in the self-consistent analysis. Before stating the physical mechanism that leads to this double-hump shape, we summarize the results obtained from the analysis of a single electronic-radiative ablation front when it is radiative enough ( $\beta_{t}$ well above unity). The expression at the leading order of the growth rate for a single ERA front reads ${ }^{8}$

$$
\gamma \approx \sqrt{k\left(g-q_{0}\left(k L_{s t}\right)^{3 / 5} \beta_{t}^{7 / 10} \mathrm{v}_{t}^{2} / L_{S t}\right)}-f_{0} \sqrt{\beta_{t}} k \mathrm{v}_{t},
$$

where $f_{0} \simeq 1.7$ and $q_{0} \simeq 3.65$. As in a single ablation front driven by thermal conduction (see Sec. III B), the ablative Rayleigh-Taylor instability in ERA fronts is mitigated by the rocket effect and the so-called convective stabilization. The rocket effect, which is usually the dominant one, is proportional to $k^{3 / 5}$, since a pure electron thermal conduction is assumed $(\nu=5 / 2)$. Regarding the convective stabilization term, it is proportional to the wavenumber, affecting, then, to the large perturbation wavenumbers. It is worth noting the stabilizing effect of radiation, which is consistent with the increased perturbed mass rate $\left(\propto \sqrt{\beta_{t}} \rho_{t} v_{t}\right)$ and dynamic pressure $\left(\propto \beta_{t}^{7 / 10} \rho_{t} v_{t}^{2}\right)$ at the ERA front. Thus, the doublehump shape, which was related to the enhancement of the coupled modes in Ref. 8, can be explained as follows: let $k_{c}^{E R A}$ be the cutoff wavenumber of the ERA front, then, any disturbance of wavelength $\delta=2 \pi / k_{c}^{E R A}$ or shorter is completely stabilized in the vicinity of the ERA front by the rocket effect mechanism. Perturbed modes (including those associated to the dynamic pressure that leads to the stabilization) are assumed to involve a region within a distance of $y^{*} \sim \delta$. Thus, the stabilizing rocket effect, which is selfgenerated by the ERA front, is felt up to a distance of $\delta$ and, if the condition $d_{p} / \delta<1$ is fulfilled, the perturbed dynamic pressure that stabilizes the ERA front will not be completely damped within the plateau region. This relaxation process will affect the RA front in the form of an additional stabilization. Since the cut-off wavelength goes like $\delta \sim \beta_{t}^{7 / 5} \mathrm{Fr}_{t}^{5 / 3}$, this additional stabilization due to coupled modes is enhanced with a higher Froude number, a higher $\beta_{t}$ (optically 


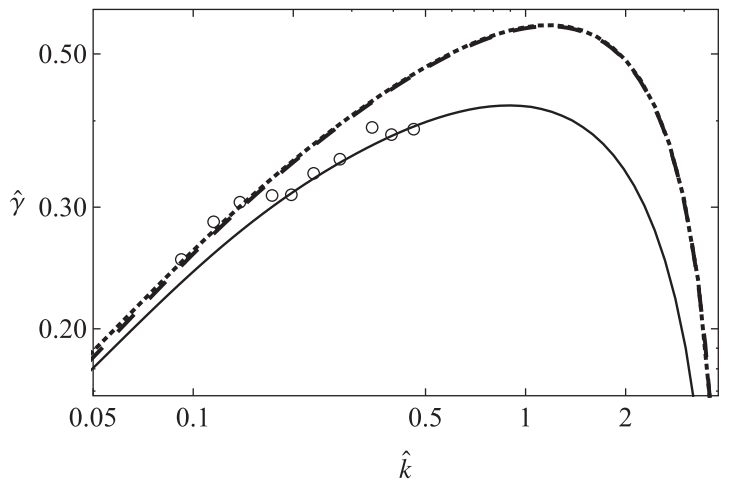

FIG. 8. Normalized growth rate for $\mathrm{SiO}_{2}$ ablator foil obtained with $2 \mathrm{D}$ single-mode simulations (circles) and applying linear theory, both, analytical sharp boundary model (dashed line) and numerical self-consistent model (solid line). Dotted line corresponds to the simplified formula derived from the SBM dispersion curve (see Appendix D). Parameters used are $r_{D}=0.22, \nu=4.7, \Delta / L_{S t}=45, d_{P} / L_{S t}=14, \beta_{t}=21$, and $\mathrm{Fr}_{\mathrm{t}}=0.7$.

thicker plateau region) or a shorter plateau length, which is consistent with the results in Ref. 8.

Figs. 8 and 9 show three comparisons of the growth rates obtained with 2D planar simulations and with the linear theory for different ablator materials and laser intensities. Singlemode 2D simulations were carried out with the radiativehydrodynamic code $\mathrm{CHIC}$, considering a $25 \mu \mathrm{m}$ layer of ablator irradiated by a laser pulse with a maximum intensity of $100 \mathrm{TW} / \mathrm{cm}^{2}$ for the cases of doped plastics (CHBr and $\mathrm{CHSi}$ ), and a $20 \mu \mathrm{m}$ layer of $\mathrm{SiO}_{2}$ subjected to a directed laser pulse with a maximum intensity of $200 \mathrm{TW} / \mathrm{cm}^{2}$. Simulation results are averaged over a $1 \mathrm{~ns}$ time duration $(1.5 \leq t \leq 2.5)$, when the target is already accelerated. Characteristic values for normalization are taken around the outer ablation front, in the point where radiative and electron temperatures are equal. An exponential regression in time is performed on the peak-to-valley perturbation depth in order to obtain an estimate of the linear growth rate (circles in the figures). Perturbation wavelengths explored cover almost a decade from $\lambda_{\min }=20 \mu \mathrm{m}$ to $\lambda_{\max }=150 \mu \mathrm{m}$. Good agreement is found between the numerical self-consistent model and the 2D planar simulations. In the glass ablator case (Fig. 8), both sharp-boundary and self-consistent models give a reasonable approximation. However, it is worth noting the cases of doped plastic (Fig. 9), where growth rates from simulations are in better agreement with the self-consistent model. This fact points out that there are some physics missing in the sharp boundary model, especially when the plateau length is of the order of the characteristic length of the RA front, $L_{0}$. Obviously, the effect of the Atwood number with a finite $L_{0}$ (that can be of the order of the plateau length) is not considered, since it is assumed a discontinuity front $\left(k L_{0} \ll 1\right)$. Another physical aspect concerns the effect of the transverse diffusion in the ablation process (Ref. 18). In the sharpboundary model for DA fronts, the transverse diffusion is taken into account in the plateau region, namely, by the thermal modes. ${ }^{8}$ However, the jump condition at the RA front related to the energy conservation law neglects it. We have taken into account the lateral thermal conduction in the energy jump condition at the RA in a similar way that in Ref. 9. The resulting dispersion relation including the effect of transverse
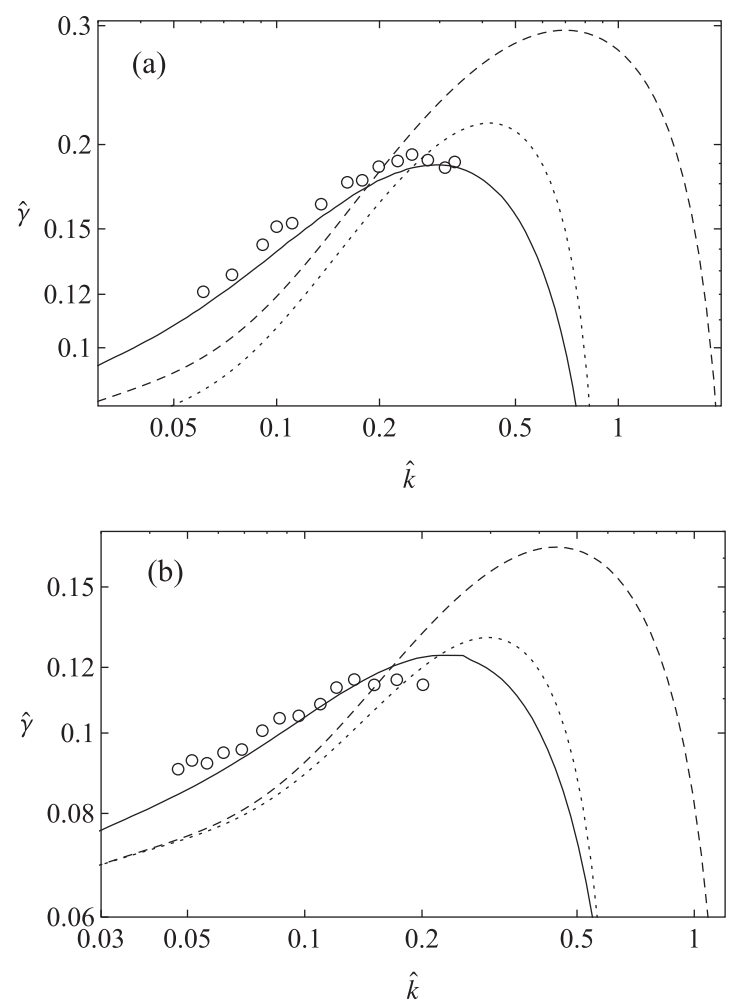

FIG. 9. Normalized growth rate obtained with 2D single-mode simulations (circles) and applying linear theory, both, analytical sharp boundary model (dashed line) and numerical self-consistent model (solid line). Dotted line corresponds to the sharp boundary model including the effects of transverse diffusion. (a) $\mathrm{CHBr}_{4 \%}$ ablator foil, parameters used are $r_{D}=0.26, \nu=1.12$, $\Delta / L_{S t}=5.2, d_{P} / L_{S t}=4.0, \beta_{t}=12.2$, and $\mathrm{Fr}_{\mathrm{t}}=0.99$. (b) $\mathrm{CHSi}_{9 \%}$ ablator foil, parameters used are $r_{D}=0.27, \nu=1.33, \Delta / L_{S t}=6.74, d_{P} / L_{S t}=5.24$, $\beta_{t}=4.5$, and $\mathrm{Fr}_{\mathrm{t}}=1.58$.

diffusion in the RA front provides a better agreement with the numerical self-consistent method in terms of the cut-off wavenumber and the maximum growth rate as it is shown in Fig. 9 .

\section{CONCLUSIONS}

We have developed a self-consistent numerical method to calculate the linear growth of perturbations in double ablation front structures due to the ablative Rayleigh-Taylor instability. Differently from the previous version of the model,${ }^{8}$ we have considered ablation fronts with a finite characteristic length. This allows us to analyze the stability of smooth hydrodynamic profiles (like those developed with doped plastics), which cannot be achieved by means of a sharp boundary model.

A radiation hydrodynamic theory is used to obtain the hydro-profiles. There, different energy transfer processes are considered: convection, electron thermal conduction, and radiation. A simplification in the $1 \mathrm{D}$ theory is possible by assuming the inner ablation front to be opaque and the outer ablation front to be transparent. This assumption leads the radiation transport to behave as a radiative thermal conduction and a cooling process, respectively. A fitting method is introduced to match theoretical hydro-profiles to those coming from one-dimensional simulations. This method uses either an error minimization procedure or takes into account the minimum density gradient scale length of both ablation 
fronts and the plateau length (outputs of the 1D simulations) in order to estimate the analytic parameters of the profiles. Comparison between the 1D theory and simulations is given showing good agreement in reproducing the profiles.

Dispersion relation is obtained numerically in the stability analysis by imposing that the perturbations are localized within the ablation region. Comparisons between the selfconsistent model and the sharp boundary one are given. It is shown that the SBM is in agreement with the self-consistent results when the characteristic lengths of both ablation fronts are sufficiently short compared to the Spitzer and the plateau length. On the contrary, if the ablation fronts are smooth, results from the two models differ, and the self-consistent dispersion relation shows an additional stabilization for short wavelengths, as it was expected. In line with SBM results, it is also observed a double-hump shape in the dispersion curves for some hydro-profiles characterized by a short plateau length. This stabilization for intermediate perturbation wavelengths is due to coupled modes; in particular, it is presumed that for a sufficiently small plateau length, the relaxation process due to the perturbed dynamic pressure generated at the outer ablation front affects the inner one for perturbation wavelengths covering the plateau region and leads to the mitigation and even to the stabilization of these modes. Some comparisons of the dispersion curve given by the selfconsistent model with growth rates obtained in 2D singlemode simulations were presented showing good agreement.
Self-consistent analysis improves the accuracy of the dispersion relation for short wavelength modes by considering ablation fronts with a finite thickness. However, the present work is based on a simple corona model where the critical density stays at the infinity, that is to say, the problem is focused within a thin layer encompassing the ablation region. Thus, physical processes occurring at distances of the order of the target radius, like inverse bremsstrahlung absorption in the low- density corona plasma, are neglected. Therefore, extensions from our work might include, besides bremsstrahlung, non-isobaric flow, spherical and unsteady effects, and multilayer shell targets, among others. These improvements may lead to more accurate linear growth rates for large wavenumbers.

\section{ACKNOWLEDGMENTS}

This work was supported by the Aquitaine Region council and by the Ministerio de Ciencia y Tecnologia of Spain (Grant No. ENE2011-28489).

\section{APPENDIX A: MATRIX OF THE LINEARIZED SYSTEM OF EQUATIONS}

For the vector of perturbed quantities defined as $\overrightarrow{\mathrm{V}}=\left(u_{1 y}, i \hat{k} u_{1 x}, p_{1}, \theta_{1}, \theta_{1}^{\prime}\right)$, the matrix of the eigenvalue problem reads

$$
\mathbf{A}^{\mathbf{R A}}=\left(\begin{array}{ccccc}
\frac{\theta_{0}^{\prime}}{\theta_{0}} & -1 & 0 & \frac{\hat{\gamma}-\theta_{0}^{\prime}}{\theta_{0}} & 1 \\
0 & -\frac{\hat{\gamma}}{\theta_{0}} & \hat{k}^{2} & 0 & 0 \\
-\frac{\hat{\gamma}+2 \theta_{0}^{\prime}}{\theta_{0}} & 1 & 0 & -\frac{F r_{t}^{-1}+\theta_{0}\left(\hat{\gamma}-2 \theta_{0}^{\prime}\right)}{\theta_{0}^{2}} & -1 \\
0 & 0 & 0 & 0 & 1 \\
\frac{\theta_{0}^{\prime}}{\theta_{0} R\left(\theta_{0}\right)} & 0 & 0 & \hat{k}^{2}+\frac{\hat{\gamma}-\theta_{0}^{\prime}}{\theta_{0} R\left(\theta_{0}\right)}-\frac{R^{\prime \prime}\left(\theta_{0}\right)}{R\left(\theta_{0}\right)} & \frac{1-2 R^{\prime}\left(\theta_{0}\right)}{R\left(\theta_{0}\right)}
\end{array}\right),
$$

where $R\left(\theta_{0}\right)=\Delta \theta_{0}^{\nu}+\theta_{0}^{5 / 2}$, and

$$
\mathbf{A}^{\mathbf{E R A}}=\left(\begin{array}{ccccc}
\frac{\theta_{0}^{\prime}}{\theta_{0}} & -1 & 0 & \frac{\hat{\gamma}-\theta_{0}^{\prime}}{\theta_{0}} & 1 \\
0 & -\frac{\hat{\gamma}}{\theta_{0}} & \hat{k}^{2} & 0 & 0 \\
-\frac{\hat{\gamma}+2 \theta_{0}^{\prime}}{\theta_{0}} & 1 & 0 & -\frac{F r_{t}^{-1}+\theta_{0}\left(\hat{\gamma}-2 \theta_{0}^{\prime}\right)}{\theta_{0}^{2}} & -1 \\
0 & 0 & 0 & 0 & 1 \\
\frac{\theta_{0}^{\prime}}{\theta_{0}^{7 / 2}} & 0 & 0 & \hat{k}^{2}+\frac{\hat{\gamma}-\theta_{0}^{\prime}}{\theta_{0}^{7 / 2}}-\frac{\left(\theta_{0}^{5 / 2}\right)^{\prime \prime}}{\theta_{0}^{5 / 2}}-\beta_{t} \frac{3 \theta_{0}^{4}-11}{2 \theta_{0}^{9}} \frac{1-2\left(\theta_{0}^{5 / 2}\right)^{\prime}}{\theta_{0}^{5 / 2}}
\end{array}\right) .
$$




\section{APPENDIX B: EXPRESSIONS OF THE MODAL ANALYSIS}

A rigorous procedure to determine the eigenmodes and their associated eigenvalues of the matrices $\mathbf{M}^{\mathbf{R A}}$ and $\mathbf{M}^{\mathbf{E R A}}$ is described in Ref. 8 .

Regarding the eigenmodes at the peak density, they can be computed with an algebraically recursive formula, that gives, for instance, $\overrightarrow{\mathbf{Y}}_{1}^{\text {left }}=\left(-1, \hat{k},\left(\hat{\gamma}-\hat{k} r_{D}\right) /\left(\hat{k} r_{D}\right), 0,1\right)$ for the eigenvalue $\lambda_{1}^{\text {left }}=\hat{k} / \hat{a}$.

At the near-corona region, the vector formal solution of the eigenmodes for an arbitrary value of $\beta_{t}$ reads, at leading order,

$$
\overrightarrow{\mathbf{Y}}^{\text {right }}(x)=x^{\lambda_{p}^{\text {right }}} \exp \left(\lambda_{0}^{\text {right }} x+\lambda_{1}^{\text {right }} x^{3 / 5}+\lambda_{2}^{\text {right }} x^{1-\alpha}\right) \overrightarrow{\mathbf{Y}}_{0}^{\text {right }},
$$

where $\psi_{0}=\left(1+\sqrt{1+4 \beta_{t}}\right) / 2$ and $\alpha=2\left(1+\beta_{t} / \psi_{0}^{2}\right) / 5$. The most unbounded eigenmode corresponds to the main eigenvalue $\lambda_{0}^{\text {right }}=\hat{k}$. The corresponding secondary eigenvalues are

$$
\begin{gathered}
\lambda_{1}^{\text {right }}=0, \\
\lambda_{2}^{\text {right }}=(2 / 5)^{\alpha} c_{1} /\left((1-\alpha) \psi_{0}^{1+\alpha}\right) \text { and } \\
\lambda_{p}^{\text {right }}=\left(1+\left(1+\sqrt{1+4 \psi_{0}^{2}}\right) /\left(2 \psi_{0}\right)\right) / 5,
\end{gathered}
$$

where $c_{1}$ is numerically computed through the expression $c_{1}=\left.\left(\theta_{0}^{3 / 2} \theta_{0}^{\prime}-\psi_{0}\right) \theta_{0}^{5 \alpha / 2}\right|_{\eta \rightarrow \infty}$.

\section{APPENDIX C: SINGLE ABLATION FRONT WITH A GENERAL SPITZER-LIKE CONDUCTIVITY (POWER LAW INDEX $v$ )}

The base flow profile is given by the equation $\theta_{0}-\theta_{0}^{\nu} \theta_{0}^{\prime}=1$, which can be integrated by defining the origin of the frame of reference, for instance, in the point where the minimum density gradient scale length takes place.

Perturbed variables are obtained by linearizing the mass and momentum conservation equation of (1) and the following energy equation:

$$
\nabla \cdot\left(\frac{5}{2} P_{0} \vec{v}-\bar{\kappa}^{*} T^{\nu} \nabla T\right)=0
$$

where $\bar{\kappa}^{*}$ is a general conductivity coefficient. By choosing the variable vector as $\overrightarrow{\mathbf{Y}}=\left(G_{1}, W_{1}, Q_{1}, T_{1}^{*}, H_{1}^{*}\right)$, where the first three components are defined in Eq. (14) and the last two ones correspond to $T_{1}^{*}=\theta_{0}^{\nu} \theta_{1}$ and $H_{1}^{*}=\left(\theta_{0}^{\nu} \theta_{1}\right)^{\prime}-u_{1 y}$, the matrix of the perturbed problem $\mathbf{Y}^{\prime}=\mathbf{M Y}$ becomes

$$
\mathbf{M}=\left(\begin{array}{ccccc}
\frac{\theta_{0}^{\prime}}{\theta_{0}} & -1 & 0 & \frac{\hat{\gamma}}{\theta_{0}^{\nu+1}} & 0 \\
-2 \hat{k}^{2} & -\frac{\hat{\gamma}}{\theta_{0}} & \hat{k}^{2} & -\frac{\hat{k}^{2}}{\theta_{0}^{\nu}} & 0 \\
-\frac{\hat{\gamma}}{\theta_{0}} & -1 & 0 & -\frac{F r_{t}^{-1}}{\theta_{0}^{\nu+2}} & 0 \\
1 & 0 & 0 & \frac{1}{\theta_{0}^{\nu}} & 1 \\
0 & 1 & 0 & \hat{k}^{2} & 0
\end{array}\right) .
$$

The most unstable mode that develops goes like $\sim y^{(3+\sqrt{5}) / 4 \nu} \exp (k y) .^{11}$

The numerical procedure to compute the growth rate is the same as described in Sec. A. Note that in this case, initial condition for the integration of the eigenvalue problem takes the form

$$
\overrightarrow{\mathbf{Y}}^{\text {left }}=\alpha_{1}\left(\theta_{0}-1\right)^{\hat{k}} \overrightarrow{\mathbf{Y}}_{1}^{\text {left }}+\alpha_{2}\left(\theta_{0}-1\right)\left(\sqrt{4 \hat{\gamma}+4 \hat{k}^{2}+1}+1\right) / 2 \overrightarrow{\mathbf{Y}}_{2}^{\text {left }} .
$$

\section{APPENDIX D: SIMPLIFIED ANALYTIC DISPERSION RELATION FORMULA FOR THE DOUBLE ABLATION FRONT}

Simplification of the DA front analytic dispersion relation formula of the sharp boundary model presented in Ref. 8 is performed under the following assumptions:

$$
\begin{aligned}
& \Delta \gg 1 \\
& \left.r_{D} \ll 1 \text { (in practice } r_{D}<0.2\right) .
\end{aligned}
$$

The dispersion relation will be built up as a matching between two asymptotic limits, small and large wavelengths compared to the plateau length $\left(\hat{k} d_{p} \gg 1\right.$ and $\hat{k} d_{p} \ll 1$, respectively). The simplified expression reads

$$
\hat{\gamma}_{D A}=\hat{\gamma}_{P}\left(1-\exp \left(-2 \hat{k} d_{p}\right)\right)+\hat{\gamma}_{S} \exp \left(-2 \hat{k} d_{p}\right)
$$

where $\hat{\gamma}_{D A}$ is the growth rate of the DA structure and $\hat{\gamma}_{P}$ and $\hat{\gamma}_{S}$ correspond to the growth rate given by each limit. Normalization is made with the values at the transition temperature of the velocity and the Spitzer length, $\mathrm{v}_{t}$ and $L_{S t} \equiv 2 \kappa_{t} T_{t} /\left(5 P_{t} \mathrm{v}_{t}\right)$, respectively.

In the first limit, the general formula is reduced to $R_{D 1} \cdot R_{D 2}=0$, where each factor is the dispersion relation of an isolated ablation front (both the inner and outer one). As the RA is much more unstable, the dispersion relation that dominates is the one associated to the first front (so, $R_{D 1}=0$ ). This is equivalent to the growth rate proposed by Piriz ${ }^{9}$ (here named as $\hat{\gamma}_{P}$ ),

$$
\hat{\gamma}^{2}+\frac{4}{1+r_{D}} \hat{k} r_{D} \hat{\gamma}-\frac{1-r_{D}}{1+r_{D}} \hat{k} \mathrm{Fr}_{\mathrm{t}}^{-1}+\hat{k}^{2} r_{D}=0
$$

In the second limit, the general formula is decomposed in a series of powers of $d_{P}$. We keep zero and first order terms. The growth rate $\hat{\gamma}_{S}$ is obtained by solving the cubic equation

$$
\begin{aligned}
\hat{\gamma}^{2} & +\bar{f}_{10}^{+} \hat{k} r_{D} \hat{\gamma}-\hat{k} \overline{\mathrm{F}} \mathrm{r}_{\mathrm{t}}^{-1}+ \\
& +C_{0} \hat{k} d_{p}\left(\hat{\gamma}^{2}+C_{1} \hat{k} r_{D} \hat{\gamma}-\hat{k}\left(C_{21} \bar{F} r_{t}^{-1}+C_{22} \mathrm{Fr}_{\mathrm{t}}^{-1}\right)\right) \\
& +\frac{4}{3} \hat{\gamma} d p\left(\hat{\gamma}^{2}+\frac{\bar{f}_{10}^{+}}{4} \hat{k} r_{D} \hat{\gamma}-\hat{k} \overline{\mathrm{F}} \mathrm{r}_{\mathrm{t}}^{-1}\right)=0,
\end{aligned}
$$

where $C_{0}=2+\bar{f}_{10}+r_{D} \bar{q}_{10}^{\prime}, \quad C_{1}=\left(2 \bar{f}_{10}^{+}+r_{D}\left({\overline{q_{10}^{\prime}}}_{10}^{\prime} 1\right)\right)$ $/ C_{0}, \quad C_{21}=\left(2+r_{D}\right) / C_{0}, \quad C_{22}=\bar{f}_{10}\left(1-r_{D}\right) / C_{0}, \quad \overline{\mathrm{Fr}}_{t}^{-1}$ $=\left(\mathrm{Fr}_{\mathrm{t}}^{-1}-\bar{q}_{10}\right), \quad \bar{f}_{10}^{+}=\bar{f}_{10}+\bar{q}_{10}^{\prime}-1, \quad \bar{f}_{10}=1.605 \sqrt{\beta_{t}}$, $\bar{q}_{10}=3.652 k^{3 / 5} r_{D} \beta_{t}^{7 / 10}$ and $q_{10}^{\prime}=1.802 \sqrt{\beta_{t}}$. 
It is worth noting the presence of the factor $\overline{\mathrm{Fr}}_{\mathrm{t}}^{-1}=$ $\left(\mathrm{Fr}_{t}^{-1}-\bar{q}_{10}\right)$ in the last expression. This reduced Froude number shows the stabilizing influence of the perturbed momentum flux. This overpressure generated on the ripply ablation surface inhibits or even completely suppresses the growth. Another important factor that we highlight is $\bar{f}_{10}^{+}=\bar{f}_{10}+\bar{q}_{10}^{\prime}-1$, which correspond to the so-called ablative stabilization. It takes an approximate value of $\bar{f}_{10}^{+} \simeq$ $3.4 \sqrt{\beta_{t}}$ for large $\beta_{t}$.

${ }^{1}$ J. D. Lindl, Phys. Plasmas 2, 3933 (1995).

${ }^{2}$ P. W. McKenty, V. N. Goncharov, R. P. J. Town, S. Skupsky, R. Betti, and R. L. McCrory, Phys. Plasmas 8, 2315 (2001); A. J. Schmitt, D. G. Colombant, A. L. Velikovich, S. T. Zalesak, J. H. Gardner, D. E. Fyfe, and N. Meztler, Phys. Plasmas 11, 2716 (2004).

${ }^{3}$ V. A. Smalyuk, S. X. Hu, V. N. Goncharov, D. D. Meyerhofer, T. C. Sangster, D. Shvarts, C. Stoeckl, B. Yaakobi, J. A. Frenje, and R. D. Petrasso, Phys. Rev. Lett. 101, 025002 (2008); T. C. Sangster, V. N. Goncharov, P. B. Radha, V. A. Smalyuk, R. Betti, R. S. Craxton, J. A. Delettrez, D. H. Edgell, V. Yu. Glebov, D. R. Harding, D. Jacobs-Perkins, J. P. Knauer, F. J. Marshall, R. L. McCrory, P. W. McKenty, D. D. Meyerhofer, S. P. Regan, W. Seka, R. W. Short, S. Skupsky, J. M. Soures, C. Stoeckl, B. Yaakobi, D. Shvarts, J. A. Frenje, C. K. Li, R. D. Petrasso, and F. H. Séguin, Phys. Rev. Lett. 100, 185006 (2008).

${ }^{4}$ V. A. Smalyuk, R. Betti. J. A. Delettrez, V. Yu. Glebov, D. D. Meyerhofer, P. B. Radha, S. P. Regan, T. C. Sangster, J. Sanz, W. Seka, C. Stoeckl, B. Yaakobi, J. A. Frenje, C. K. Li, R. D. Petrasso, and F. H. Séguin, Phys. Rev. Lett. 104, 165002 (2010).

${ }^{5}$ S. Fujioka, A. Sunahara, K. Nishihara, N. Ohnishi, T. Johzaki, H. Shiraga, K. Shigemori, M. Nakai, T. Ikegawa, M. Murakami, K. Nagai, T. Norimatsu, H. Azechi, and T. Yamanaka, Phys. Rev. Lett. 92, 195001 (2004); S. Fujioka, A. Sunahara, N. Ohnishi, Y. Tamari, K. Nishihara, H. Azechi,
H. Shiraga, M. Nakai, K. Shigemori, T. Sakaiya, M. Tanaka, K. Otani, K. Okuno, T. Watari, T. Yamada, M. Murakami, K. Nagai, T. Norimatsu, Y. Izawa, S. Nozaki, and Y.-W. Chen, Phys. Plasmas. 11, 2814 (2004).

${ }^{6}$ J. Sanz, R. Betti, V. A. Smalyuk, M. Olazabal-Loumé, V. Drean, V. Tikhonchuk, X. Ribeyre, and J. Feugeas, Phys. Plasmas 16, 082704 (2009).

${ }^{7}$ V. Drean, M. Olazabal-Loumé, J. Sanz, and V. T. Tikhonchuk, Phys. Plasmas 17, 122701 (2010)

${ }^{8}$ C. Yañez, J. Sanz, M. Olazabal-Loumé, and L. F. Ibañez, Phys. Plasmas 18, 052701 (2011)

${ }^{9}$ A. R. Piriz, J. Sanz, and L. F. Ibañez, Phys. Plasmas 4, 1117 (1997).

${ }^{10}$ J. Sanz, Phys. Rev. Lett. 73, 2700 (1994); J. Sanz, Phys. Rev. E 53, 4026 (1996).

${ }^{11}$ R. Betti, V. Goncharov, R. L. McCrory, and C. P. Verdon, Phys. Plasmas 2, 3844 (1995); V. N. Goncharov, R. Betti, R. L. McCrory, P. Sorotokin, and C. P. Verdon, Phys. Plasmas 3, 1402 (1996); V. N. Goncharov, R. Betti, R. L. McCrory, and C. P. Verdon, Phys. Plasmas 3, 4665 (1996).

${ }^{12}$ H. J. Kull, Phys. Fluids B 1, 170 (1989).

${ }^{13}$ R. Betti, V. N. Goncharov, R. L. McCrory, and C. P. Verdon, Phys. Plasmas 5, 1446 (1998).

${ }^{14}$ J. Breil and P. H. Maire, J. Comput. Phys. 224, 785 (2007); P. H. Maire, R. Abgrall, J. Breil, and J. Ovadia, SIAM J. Sci. Comput. 29, 1781 (2007); P. H. Maire, J. Comput. Phys. 228, 2391 (2009).

${ }^{15}$ Y. B. Zel'dovich and Y. P. Razier, Physics of Shock Waves and High-Temperature Hydrodynamic Phenomena (Academic, New York, 1966).

${ }^{16}$ D. G. Hicks, B. K. Spears, D. G. Braun, R. E. Olson, C. M. Sorce, P. M. Celliers, G. W. Collins, and O. L. Landen, Phys. Plasmas 17, 102703 (2010).

${ }^{17} \mathrm{~S}$. Atzeni and J. Meyer-Ter-Vehn, The Physics of Inertial Fusion (Oxford University Press, Oxford, 2004).

${ }^{18}$ P. Clavin and L. Masse, Phys. Plasmas 11, 690 (2004); J. Sanz, L. Masse, and P. Clavin, Phys. Plasmas 13, 102702 (2006). 
Physics of Plasmas is copyrighted by the American Institute of Physics (AIP). Redistribution of journal material is subject to the AIP online journal license and/or AIP copyright. For more information, see http://ojps.aip.org/pop/popcr.jsp 